\title{
Small GTPase Cdc42 Is Required for Multiple Aspects of Dendritic Morphogenesis
}

\author{
Ethan K. Scott, John E. Reuter, and Liqun Luo \\ Department of Biological Sciences, Stanford University, Stanford, California 94305-5020
}

\begin{abstract}
The study of dendritic development in CNS neurons has been hampered by a lack of complex dendritic structures that can be studied in a tractable genetic system. In an effort to develop such a system, we recently characterized the highly complex dendrites of the vertical system (VS) neurons in the Drosophila visual system. Using VS neurons as a model system, we show here using loss-of-function mutations that endogenous $\mathrm{Cdc} 42$, a member of Rho family of small GTPases, is required for multiple aspects of dendritic morphogenesis. Cdc42-mutant VS neurons display normal complexity but increased dendritic length compared with wild type and have defects in dendrite caliber and stereotyped dendritic branch positions. Remarkably, $C d c 42$ mutant neurons also show a 50\% reduction in dendritic spine density. These results demonstrate that $\mathrm{Cdc} 42$ is a regulator for multiple aspects of dendritic development.
\end{abstract}

Key words: Drosophila; vertical system; dendrites; dendritic spines; Rho GTPases; actin cytoskeleton

\section{Introduction}

The complex and characteristic structures of dendrites are a crucial part of the neuronal architecture that underlies brain function; as such, their development has been a focal point of recent research (for review, see McAllister, 2000; Cline, 2001; Jan and Jan, 2001; Scott and Luo, 2001). It is generally believed that dendritic morphogenesis requires an intrinsic differentiation program that is further instructed by extracellular cues and electrical activity. Eventually, these different forces converge to control cytoskeletal dynamics that specify dendritic growth, branching, and the formation of dendritic spines (Scott and Luo, 2001).

The Rho family of small GTPases, notably RhoA, Rac, and $\mathrm{Cdc42}$, are key regulators of the actin cytoskeleton in response to extracellular cues (Etienne-Manneville and Hall, 2002). In the context of neuronal development, an increasing number of key extracellular cues known to regulate neuronal morphogenesis have been linked to the regulation of Rho GTPases, as have neurotransmitter receptors that mediate activity-dependent dendritic morphogenesis. Well characterized signaling pathways from Rho to actin identified in non-neuronal cells have also started to be characterized in neurons (for review, see Luo, 2002). Not surprisingly, perturbation of activities of Rho GTPases themselves by expression of dominant-negative or constitutively active versions of different Rho GTPases often leads to dramatic effects in neuronal morphogenesis (for review, see Luo, 2000; Redmond and Ghosh, 2001). A general consensus has emerged from these studies: Rac and Cdc42 have generally been thought to promote process growth and attractive guidance, whereas RhoA activation leads to retraction or repulsive guidance (Jalink et al., 1994; Luo

Received Aug. 15, 2002; revised Jan. 23, 2003; accepted Jan. 29, 2003.

This work was supported by National Institutes of Health Grants R01-NS36623 (L.L.) and TR32-HD07249 (E.K.S.). We thank R. Fehon for providing Cdc42 mutants and G. Jefferis and A. Goldstein for their comments on this manuscript.

Correspondence should be addressed to Liqun Luo at the above address. E-mail: Iluo@stanford.edu. Copyright $\odot 2003$ Society for Neuroscience $\quad 0270-6474 / 03 / 233118-06 \$ 15.00 / 0$ et al., 1994; Kozma et al., 1997; Threadgill et al., 1997; Ruchhoeft et al., 1999; Li et al., 2000; Nakayama et al., 2000; Wong et al., 2000). However, notable exceptions have made these interpretations far from conclusive (Jin and Strittmatter, 1997; Bashaw et al., 2001).

Studies involving dominant mutant expression, although informative, have a number of caveats. Dominant-negative Rho GTPase mutants are believed to act by titrating guanine nucleotide exchange factors (Ridley et al., 1992). If two Rho GTPases share a common exchange factor (most exchange factors have activities toward several Rho GTPases, at least in vitro), then expressing a dominant-negative mutant of one GTPase (e.g., Rac) could interfere with the function of another GTPase (e.g., Cdc42). Likewise, because some Rho GTPases share downstream effectors, overexpressing constitutively active form of one GTPase could also cross-activate other GTPases. For instance, certain roles assigned to Rac from dominant-negative mutant studies have not been reproduced using null mutations (HakedaSuzuki et al., 2002), suggesting that cross- inhibition-activation of other Rho family GTPases might be a cause for the effects seen in dominant mutant-expressing cells. In addition, the expression of dominant mutants bypasses normal regulation of these GTPases. In summary, dominant mutant studies have limitations in defining roles of individual GTPases and fine aspects of their regulation.

We therefore sought to evaluate the function of endogenous Rho GTPases in dendritic morphogenesis using loss-of-function mutants. Because of the ubiquitous function of Rho GTPases in development, we developed a genetic mosaic method by which we could generate a very small population of uniquely labeled homozygous mutant neurons in an otherwise heterozygous and unlabeled brain (Lee and Luo, 1999). Using this method termed MARCM (for mosaic analysis with a repressible cell marker) and focusing on Drosophila mushroom body (MB) neurons, we found that RhoA mutants extend their dendrites beyond wild- 
type confines (Lee et al., 2000), whereas triple mutants for three Rac genes exhibit a $\sim 50 \%$ reduction in total dendritic length and branching number ( $\mathrm{Ng}$ et al., 2002). Here we describe our study of the function of $\mathrm{Cdc} 42$ in dendritic morphogenesis using lossof-function mutants and compare our finding with previous studies of Rac and Rho in Drosophila and mammalian neurons.

\section{Materials and Methods}

Drosophila were grown on standard media at $25^{\circ} \mathrm{C}$. During clonal analysis, larvae hatched over a $2 \mathrm{hr}$ interval were moved to vials containing 10 $\mathrm{ml}$ of food and kept at a concentration of 80 per vial. Mitotic recombination was induced via heat shock $\left(40 \mathrm{~min}\right.$ in a $37^{\circ} \mathrm{C}$ water bath, $30 \mathrm{~min}$ at room temperature, and $40 \mathrm{~min}$ in $37^{\circ} \mathrm{C}$ water bath) at 2 and $3 \mathrm{~d}$ after hatching. Adult female flies between 2 and $5 \mathrm{~d}$ after eclosion were dissected, fixed, and stained as described previously (Lee et al., 1999).

A Bio-Rad (Hercules, CA) MRC 1024 laser scanning confocal microscope and the Laser Sharp image collection program were used. Images were prepared using Adobe Photoshop (Adobe Systems, San Jose, CA). Three-dimensional traces of the dendrites were produced from confocal stacks using MicroBrightField (Colchester, VT) Neurolucida software (Scott et al., 2002). Briefly, dendritic branches were traced such that turning points, branch points, and endpoints were specified in $X, Y$, and $Z$ positions within the confocal stack. MicroBrightField NeuroExplorer software was then used to give quantitative measures. Branching complexity was measured as the total number of branch points in the dendritic tree, and total length for these traces was defined as the combined length of all of the branch segments. In quantifying spines, we included terminal segments from 1 to $3 \mu \mathrm{m}$ long, because these were the structures with the most dense postsynaptic terminals as described previously using electron microscopy (EM) (Hausen et al., 1980). Our efforts to develop a postsynaptic marker as a means of quantifying spines were unsuccessful; thus we used staining from membrane-localized CD8-green fluorescent protein (GFP) to identify these spines. Structures longer than $3 \mu \mathrm{m}$ were treated as dendritic branches.

\section{Results}

Loss-of-function Cdc42 mutants (Fehon et al., 1997) exhibit a number of developmental abnormalities, including subtle defects of longitudinal commissures in the Drosophila embryonic nervous system (Genova et al., 2000). Their effects on dendritic development have not been described. Our preliminary MARCM analysis suggests that $C d c 42$ mutants have no detectable effects on dendritic morphogenesis of MB neurons (our unpublished data), possibly because dendrites from individual $\mathrm{MB}$ neurons are simple and variable. We therefore sought to test Cdc42 function in dendritic morphogenesis using a more complex and stereotyped dendritic tree. We recently characterized lobula plate giant neurons that possess complex and stereotyped dendritic trees (Scott et al., 2002). Here we first describe and further characterize one of these neurons, vertical system neuron 1 (VS1), and then use this model neuron to investigate the function of Cdc42 using lossof-function mutants.

\section{The dendritic tree of the VS1 neuron}

There are six vertical system cells in each Drosophila lobula plate that bear a close structural resemblance to the well characterized VS neurons in blowflies and house flies (Pierantoni, 1976; Strausfeld, 1976; Eckert and Bishop, 1978; Hausen et al., 1980; Hengstenberg et al., 1982). Each cell has a complex elaboration of dendrites in the lobula plate with axons that travel medially and terminate near the esophagus (Fig. $1 A$ ).

Because different neurons of the vertical system have different characteristic structures and levels of complexity (Scott et al., 2002), it was necessary to select a single type of VS neuron for quantitative analyses. We restricted our quantitative analyses to
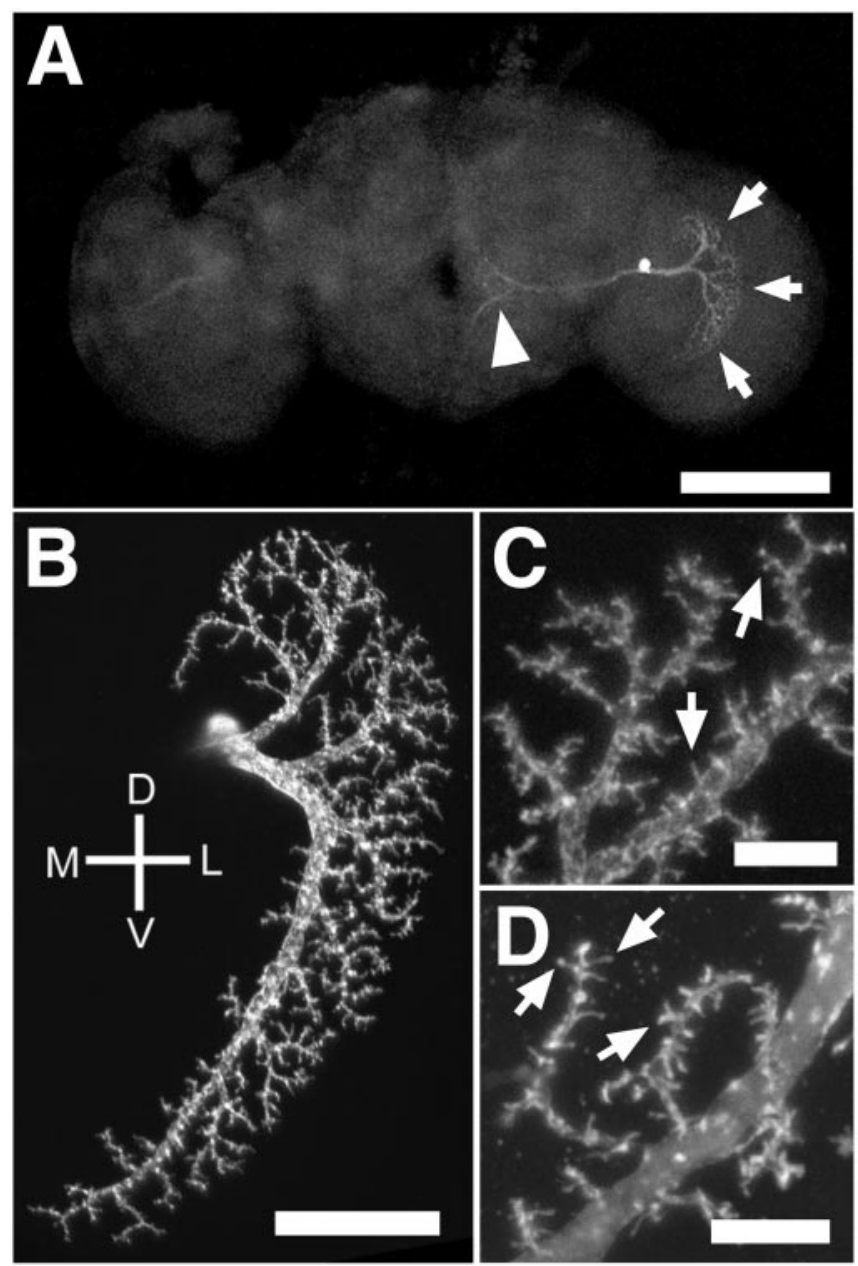

Figure 1. Structure of the VS1 neuron. The position of a VS1 cell is shown in the context of the whole Drosophila brain by the MARCM labeling method $(A)$. The dendrites can be seen in the Iobula plate (arrows), and the axon extends medially (arrowhead), terminating near the midline. A close-up view of the dendrites of a VS1 cell ( $B$ ) shows a major dendritic shaft that sweeps from dorsal to ventral. Smaller branches leave this shaft to form a dendritic field that covers that lateral portion of the lobula plate. A closer view of the dendrites of a different VS1 clone (C) shows small spine-like structures (arrows). Like vertebrate spines, these structures are rich in actin, as evidenced by dendrites with spines brightly stained against actin-GFP (arrows; $D$ ). Scale bars: $A, 100 \mu \mathrm{m} ; B, 25 \mu \mathrm{m} ; C, D, 5 \mu \mathrm{m}$. D, Dorsal; V, ventral; $M$, medial; $L$, lateral. Wild-type flies in A-Care hs-flp/+;FRT ${ }^{G 13}$, tubP-GAL80/FRT ${ }^{G 13}$, UAS-mCD8-GFP; GAL4-3A/+ or hs-flp, UAS-mCD8-GFPI+; tubP-GAL80, FRT $T^{2 A} / G A L 4-3 A, F R T^{2 A}$. Genotype for $D$ is tubPGAL80, hs-flp, FRT $T^{19 A} / y w, F R T^{19 A} ;$ UAS-GFP-actin, UAS-myc-tubulin/+;GAL4-3A/+.

the VS1 neuron because it is unambiguously recognizable, highly stereotyped, and has the most complex dendrites of any VS neuron (Fig. $1 A, B$ ) (Scott et al., 2002). The VS1 dendrite is characterized by a main dendritic shaft that produces one or a few dorsally projecting branches before sweeping ventrally. As the main shaft extends ventrally, it continues to produce smaller branches that combine to form a narrow band covering the lateral part of the lobula plate (Fig. $1 B$ ). All findings for VS1 neurons described below, at least at the qualitative level, also apply to other classes of VS neurons (data not shown).

To define quantitatively some aspects of the structure of the dendrites, we first obtained three-dimensional confocal images of VS1 dendritic trees and then traced the dendrites to produce three-dimensional computer diagrams of the dendrites. From these tracings, we measured dendritic branching complexity on the basis of the total number of branch points found in the den- 
Table 1. The effect of Cdc42 mutation on VS1 neurons

\begin{tabular}{lccc}
\hline & WT (12 hr light/dark) & Cdc42 & Cdc42 $2^{4}+$ UAS-Cdc42 \\
\hline Number of dendritic branch points & $158.1 \pm 8.7(13)$ & $154.3 \pm 5.0(9)$ & $157.8 \pm 3.4(4)$ \\
Total length of dendrites $(\mu \mathrm{m})$ & $975 \pm 37(10)$ & $1135 \pm 43(9)^{* *}$ & $1199 \pm 15(4)^{* * *}$ \\
Number of spines & $387 \pm 21(8)$ & $230 \pm 17(9)^{* * *}$ & $225 \pm 21(4)^{* * *}$ \\
Spine density $($ spines $/ \mu \mathrm{m})$ & $0.418 \pm 0.034(8)$ & $0.203 \pm 0.012(9)^{* * *}$ & $0.187 \pm 0.016(4)^{* * *}$ \\
Number of axon branch points & $5.11 \pm 0.61(9)$ & $8.30 \pm 0.67(10)^{* *}$ & $9.25 \pm 1.31(4)^{*}$ \\
Penetrance of dendrite caliber inconsistency & $0 / 13$ & $3 / 10$ & $0 / 7$ \\
Penetrance of medially shifted branch & $0 / 13$ & $5 / 10$ & $0 / 7$ \\
\hline
\end{tabular}

Values are shown \pm SEM, with experimental $n$ in parentheses. $t$ test was used to measure statistical significance compared with wild type (WT). ${ }^{*} p<0.05 ;{ }^{* *} p<0.01 ;{ }^{* * *} p<0.001 ; 0$ others, no statistical difference. $n$ varies within genotype because different measures require different image quality. All quantitative analyses were restricted to VS1 neurons. Our analysis of the penetrance of qualitative defects includes VS1 and VS2 neurons.

drites of single VS1 cells. We also used the tracings to determine the combined length of all of the dendritic branches for each cell (Table 1).

\section{Dendritic spines of the VS1 neuron}

Of special interest in these VS neurons is the presence of structures that resemble dendritic spines found in many vertebrate neurons (Fig. 1C, arrows). In vertebrates, dendritic spines represent the locations of excitatory synapses (for review, see Bonhoeffer and Yuste, 2002). Similarly, in the blowfly Calliphora erythrocephala and the housefly Musca domestica, studies using EM of these analogous VS neurons have shown that these spine-like structures are enriched for postsynaptic structures (Pierantoni, 1976; Hausen et al., 1980). The fact that EM studies in two different species of Dipteran insect have shown postsynaptic densities in the spine-like structures, along with the similarity of these structures among Dipteran insects
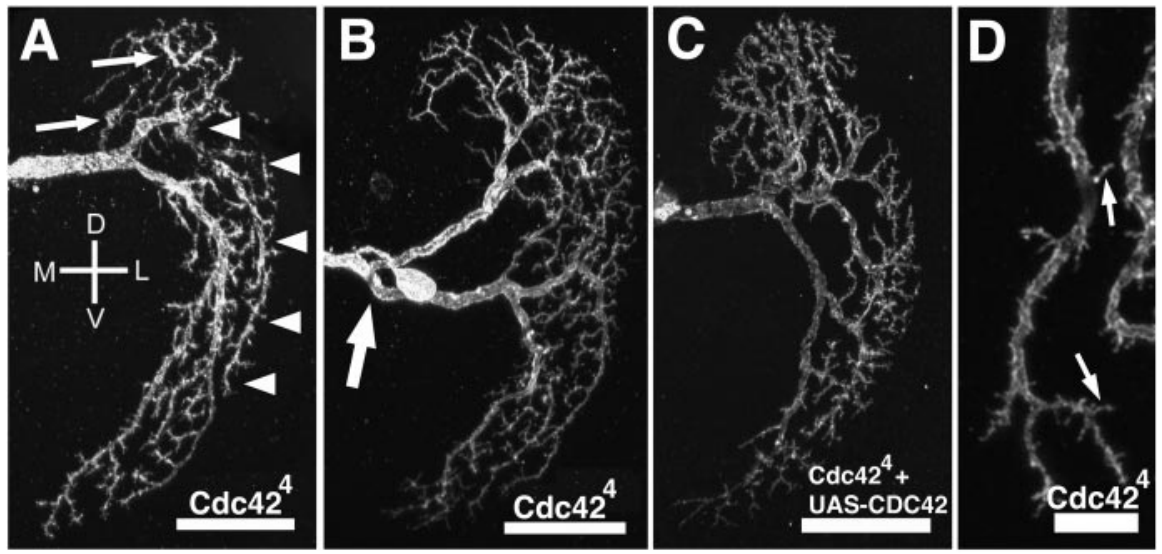

Figure 2. Dendritic phenotypes in C $d c 42$ mutant neurons. VS1 cells homozygous for $C d c 42^{4}$ show defects in dendrite caliber, in which distal dendrites are thicker than proximal dendrites ( $A$; arrows), and misguided dendrites, such as one that innervates the ventral region of the dendritic field despite its originating from a dorsal branch ( $A$; arrowheads). Another $C d c 42$ mutant neuron shows a first branch that is shifted medially ( $B$; arrow). For comparison with a normally positioned first branch, see Figure $1 B$, or $A$ and $C$ in this figure. Qualitative phenotypes are rescued in $C d c 42^{4}$ mutant cells with a UAS-Cdc42 transgene $(C)$. A close-up view of a Cdc42 mutant VS1 dendrite (D) shows fewer spines (arrows) compared with wild type (Fig. $1 C, D)$. Scale bars: $A-C, 25 \mu \mathrm{m} ; D, 5$ $\mu \mathrm{m}$. D, Dorsal; V, ventral; M, medial; L, lateral. Cdc42 mutant flies are tubP-GAL80, hs-flp, FRT $T^{19 A} / y w, C d c 42^{4}, F R T^{19 A} ;$ UAS-mCD8GFPI+; GAL4 -3A/+ for $A, B$, and D. Rescue flies are tubP-GAL80, hs-flp, FRT $T^{19 A} / y w, C d c 42^{4}, F R T^{19 A}$; UAS-mCD8-GFPI+; GAL4 -3A/UAS-CdC42 in $C$. as observed by light microscopy (Strausfeld, 1976; Eckert and Bishop, 1978; Hengstenberg et al., 1982) (Fig. $1 D$ ), suggests that all of these insects probably use these spine-like structures as postsynaptic terminals.

Another characteristic feature of vertebrate dendritic spines is that they are actin-based structures, whose changes in morphology may be important for synaptic plasticity (Bonhoeffer and Yuste, 2002). We were interested in determining whether these vertical system spines are similar to their vertebrate counterparts in cytoskeletal structure. To this end, we expressed an actin-GFP fusion protein (Verkhusha et al., 1999) in single VS1 cells using the MARCM system (Lee and Luo, 1999). We found that this actin-GFP is present robustly in the VS1 spines (Fig. 1D), indicating that actin is likely the important cytoskeletal element of these structures.

Together with the EM studies from larger insects summarized above, we propose that these spine-like structures in insect VS cells are similar to vertebrate dendritic spines and will refer to them as spines hereafter for simplicity. Future morphological and physiological studies are needed to determine how analogous these spines are to their vertebrate equivalent.

Cdc42 mutants affect dendritic caliber consistency, branching pattern, and dendritic spine density

Having characterized wild-type VS1 dendritic branching patterns and dendritic spines, we compared them with those in single-cell MARCM clones in which the labeled VS1 cells are homozygous

for loss-of-function mutants of $C d c 42$. Most experiments reported here made use of the $C d c 42^{4}$ allele, which contains a mutation in a consensus splice acceptor that leads to strong loss-offunction (Fehon et al., 1997). The phenotypes seen in $C d c 42^{4}$ clones were also found in clones homozygous for another strong loss-of-function allele, $C d c 42^{3}$ (Fehon et al., 1997), but not for the control FRT chromosome (data not shown).

VS1 neurons homozygous for $C d c 42^{4}$ show a variety of defects compared with wild type (Figs. $2 A, B, D, 3 A, B$ ). Whereas the dendrites of wild-type VS cells taper smoothly from thick near the base to thin at distal tips (Fig. $1 \mathrm{~B}$ ), the caliber of $C d c 42^{4} \mathrm{VS}$ cells is occasionally inconsistent (Table 1 ). Some dendrites are thinner near their major branches than in more distal positions (Fig. $2 \mathrm{~A}$, arrows). Also, the branching pattern of the dendrites is often abnormal. In wild-type VS1 cells, major branches off of the main dendritic shaft send their smaller dendrites only to the region near the original branch. A dendritic tip in the ventral part of the field, for instance, would be derived from a major branch off of the ventral part of the main shaft (Fig. $1 B$ ). In $C d c 42$ mutant cells, dendritic tips may be derived from major branches in distant parts of the field (Fig. $2 \mathrm{~A}$, arrowheads). One branch in particular, the most medial branch in the dendritic tree, is often shifted in $C d c 42^{4}$ cells (Table 1). In wild-type VS1 neurons, the initial branches extend almost directly dorsally, departing from the main branch near where it begins to turn ventrally (Scott et al., 2002) (Fig. 1B). The medial shift observed in Cdc42 mutant cells 

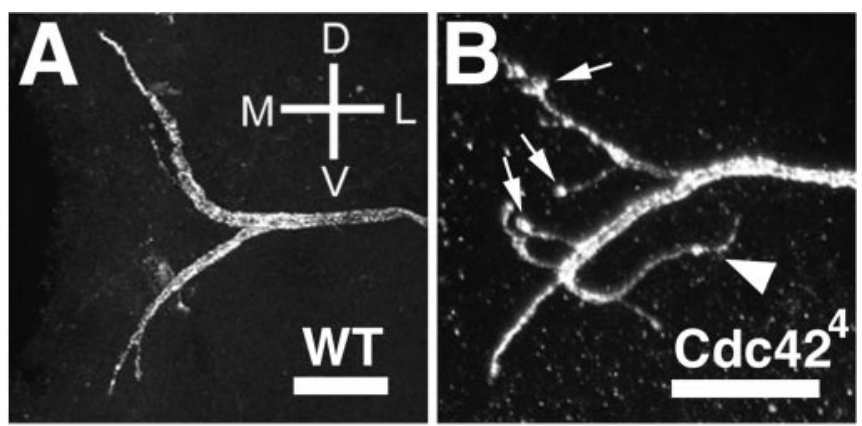

Figure 3. Axonal phenotypes in C $d$ c42 mutant neurons. The axonal terminus of a wild-type VS1 neuron is shown in $A$. The axon splits into two major branches, each of which may have one or a few additional small branches. All branches remain in the vicinity of the medially extending major branches. The axonal terminus of a $C d c 42^{4} \mathrm{VS} 1$ neuron is shown in $B$. There is an increase in the number of branches, the termini of the axons are often tipped with enlarged structures (arrows), and axons occasionally turn laterally (arrowhead), away from the midline. Scale bars, $10 \mu \mathrm{m}$. D, Dorsal; V, ventral; M, medial; L, lateral.

results in a more pronounced split between the dorsal and ventral halves of the dendritic field (Fig. 2B, arrow). Quantitatively, whereas the dendritic branching complexity of wild-type and $C d c 42^{4}$ VS1 neurons is similar, $C d c 42^{4}$ VS1 neurons had a significantly greater overall dendritic length (Table 1).

Perhaps the most remarkable defect in $C d c 42$ mutant neurons is the reduction of the numbers of well developed dendritic spines (compare Fig. $2 A, B$ with Fig. $1 B$ ). At high magnification (compare Fig. $2 D$ with Fig. $1 C, D$ ), we did observe spines that are similar to wild type, but these spines are not as evenly spaced and the density is much reduced. Using a uniform definition of regarding protrusions between 1 and $3 \mu \mathrm{m}$ as spines (see Materials and Methods), we recorded a 50\% reduction in Cdc42 mutant VS1 cells compared with wild-type control (Table 1).

\section{Axon defects in $C d c 42$ mutants}

Wild-type VS1 axons project medially, with stereotyped branching pattern and terminal fields (Figs. $1 A, 3 A$ ). This pattern is disrupted in $C d c 42^{4}$ VS1 cells, some of which exhibit misguided axons that turn laterally, away from their wild-type target field (Fig. 3B, arrowhead). Additionally, the axons of $C d c 42^{4}$ neurons form more branches in this terminal field than do their wild-type counterparts (Table 1) and often exhibit enlarged terminals rarely observed in wild type (Fig. $3 B$, arrows).

\section{Attempts to assess cell autonomy of Cdc42 action}

In the MARCM strategy, all labeled clones are homozygous mutant; however, some homozygous mutant cells may not be labeled because they do not express the GAL4 line used to visualize the clone. Because the expression of the GAL4-3A driver is primarily restricted to the lobula plate giant neurons, it remains possible that some phenotypes we observe in $C d c 42^{4}$ MARCM clones are attributable to disruption of Cdc42 function in as yet uncharacterized neurons presynaptic or postsynaptic to VS1, or glia. To determine the degree to which the phenotype is cell autonomous, we expressed UAS-Cdc42 transgenes in $C d c 42^{4}$ MARCM clones, such that wild-type Cdc42 is resupplied only to the labeled neurons. An important caveat to this experiment is that the transgene is not immediately expressed after generation of the clone. The GAL80 repressor protein must be sufficiently diluted, which can take a long time in single-cell clones, to allow GAL4-induced gene expression. For instance, we could not observe reliable marker expression in VS1 until very late pupa, well after the initial den- dritic morphogenesis has already taken place (data not shown). Indeed, many of the quantitative defects in $C d c 42^{4}$ cells are not affected by the UAS-Cdc42 transgene (Table 1). Nevertheless, certain qualitative defects, such as dendritic caliber or branching pattern, appear to be rescued by the expression of the wild-type Cdc42 transgene (Fig. 2C, Table 1), indicating that Cdc42 function in at least these aspects of dendritic morphogenesis is cell autonomous. The failure to rescue dendritic length or spine density defects could be caused, strictly speaking, by the nonautonomous effect of Cdc42. More likely, it is a result of inadequate expression at a time when $\mathrm{Cdc} 42$ is required, implying that controlling dendritic length (likely a consequence of dendritic branch misguidance) and spine formation may require a higher amount of Cdc42 than controlling initial branch formation and caliber consistency.

\section{Discussion}

Previous studies using dominant-negative and constitutively active Cdc42 mutants have implicated Cdc42 in a variety of functions relating to dendritic growth, branching, and branch stability (Luo et al., 1994; Threadgill et al., 1997; Gao et al., 1999; Ruchhoeft et al., 1999; Li et al., 2000). However, the effects of dominant Cdc42 and Rac are generally not distinguishable (Threadgill et al., 1997; Gao et al., 1999; Li et al., 2000). Because Rac GTPases are required for dendritic growth and branching from loss-of-function studies ( $\mathrm{Ng}$ et al., 2002), it is possible that some of the dominant $\mathrm{Cdc} 42$ phenotypes are caused by crossinhibition or activation of Rac GTPases. In this study, we provide definitive evidence that $\mathrm{Cdc} 42$ is required for dendritic and axonal morphogenesis using loss-of-function mutants. What new aspects of Cdc42 function have we learned from these loss-offunction phenotypic analyses?

Compared with mutants defective in Rac GTPases (Ng et al., 2002), Cdc42 mutants have much less profound effects. In fact, we could not observe any significant dendritic and axonal $C d c 42$ mutant phenotypes in single-cell clones of MB neurons (data not shown) in which mutants of Rac GTPases exhibit drastic effects (Ng et al., 2002). This is not because Cdc42 is not expressed in mushroom bodies; in fact, in MB neuroblast clones homozygous for $C d c 42$ mutations, we did observe a $100 \%$ penetrant defect in neuroblast proliferation (our unpublished observation). Because the dendrites of MB neurons are simple and highly variable, we made use of VS1 neurons in which the dendritic tree is much more complex and stereotyped. Even in VS1 neurons, the gross organization of dendritic tree is quite normal in $C d c 42$ mutants; the total dendritic tree length is even increased compared with control, contrary to the general notion that $\mathrm{Cdc} 42$ promotes neuronal process growth. However, this effect could be the product of misguided dendritic branches (see below) that may not innervate the dendritic field as efficiently as their wild-type counterparts.

Despite having grossly normal dendritic trees, Cdc42 mutant VS1 cells do display a number of important defects in dendritic morphogenesis. First, dendritic caliber consistency is disrupted. A universal property of dendritic trees is that they taper smoothly from thick near the base to thin at distal tips. To our knowledge, this is the first mutant that has been described to disrupt this property, suggesting that $\mathrm{Cdc} 42$ activity is necessary for regulating dendritic caliber diameter, an aspect that may be important for integration of synaptic potential within the dendrites (Jan and Jan, 2001). 
Second, although the gross dendritic branching complexity is not affected, the stereotyped branching pattern is disrupted (Table 1). This defect may reflect abnormal responses of the dendrites to branching signals at key locations during development, consistent with the idea that $\mathrm{Cdc} 42$ transduces extracellular signals to regulate dendritic branching rather than being required for the cell biology of branching per se.

Third, $C d c 42$ mutation has a drastic effect on dendritic spine development: it reduces dendritic spine number to approximately one-half of that of wild type. It remains possible that the reduction in number is in fact reduction in size, such that the smaller-sized "spines" are no longer quantified as spines by our criterion. Given the small size of even the wild-type dendritic spines approaching the resolution limit of light microscopy, it is currently difficult to distinguish these possibilities. Whatever the mechanism is, our observation underscores the importance of endogenous $\mathrm{Cdc} 42$ in spine development. Previous studies in mammalian neurons using dominant mutant expression have implicated the function of Rac and Rho in spine development (Luo et al., 1996; Nakayama et al., 2000; Tashiro et al., 2000) (for review, see Bonhoeffer and Yuste, 2002); our study now provides evidence for the involvement of Cdc42 in spine morphogenesis. Given the actin-rich nature of dendritic spines and requirement of actin polymerization in spine morphogenesis and motility (Fischer et al., 1998; Dunaevsky et al., 1999; Zhang and Benson, 2001), it is perhaps not surprising that a major regulator of actin polyermization such as $\mathrm{Cdc} 42$ should play an important role.

In summary, our data demonstrate a genetic requirement for Cdc42 in certain aspects of VS1 dendrite development, including regulating dendritic branching, guidance, caliber consistency, and dendritic spine density. Given the function of Cdc42 in regulating actin polymerization (Etienne-Manneville and Hall, 2002), these phenotypes may be a consequence of abnormal communication between extracellular factors and actin-rich structures such as filopodia, growth cones, and spines, leading to errors in branching, growth, or guidance of developing dendrites. Our study also demonstrates the utility of the vertical system (and VS1 specifically) as a model system for dendritic studies. One drawback of the current system for assessing cell autonomy is the potential for incomplete rescue resulting from GAL4/UASmediated transgene expression. In future studies, stronger and earlier-expressing GAL4 lines in these neurons may overcome this problem. Nonetheless, the dendrites of these cells are highly complex and stereotyped and provide the advantage of having spines structurally similar to those in vertebrates. Access to these model cells in Drosophila allows for the study of complex dendrites in a highly tractable genetic system. We believe that this will prove particularly useful in characterizing phenotypes for candidate genes and thereby defining their roles in dendritic morphogenesis.

\section{References}

Bashaw GJ, Hu H, Nobes CD, Goodman CS (2001) A novel Dbl family RhoGEF promotes Rho-dependent axon attraction to the central nervous system midline in Drosophila and overcomes Robo repulsion. J Cell Biol 155:1117-1122.

Bonhoeffer T, Yuste R (2002) Spine motility: phenomenology, mechanisms, and function. Neuron 35:1019-1027.

Cline HT (2001) Dendritic arbor development and synaptogenesis. Curr Opin Neurobiol 11:118-126.

Dunaevsky A, Tashiro A, Majewska A, Mason C, Yuste R (1999) Developmental regulation of spine motility in the mammalian central nervous system. Proc Natl Acad Sci USA 96:13438-13443.
Eckert HE, Bishop LG (1978) Anatomical and physiological properties of the vertical cells in the third optic ganglion of Phaenicia sericata (Diptera, Calliphoridae). J Comp Physiol 126:57-86.

Etienne-Mannville S, Hall A (2002) Rho GTPase in cell biology. Nature 420:629-635.

Fehon R, Oren T, LaJeunesse DR, Melby TE, McCartney BM (1997) Isolation of mutations in the Drosophila homologues of the human Neurofibromatosis 2 and yeast $C D C 42$ genes using a simple and efficient reversegenetic method. Genetics 146:245-252.

Fischer M, Kaech S, Knutti D, Matus A (1998) Rapid actin-based plasticity in dendritic spines. Neuron 20:847-854.

Gao FB, Brenman JE, Jan LY, Jan YN (1999) Genes regulating dendritic outgrowth, branching, and routing in Drosophila. Genes Dev 13:2549-2561.

Genova JL, Jong S, Camp JT, Fehon RG (2000) Functional analysis of Cdc42 in actin filament assembly, epithelial morphogenesis, and cell signaling during Drosophila development. Dev Biol 221:181-194.

Hakeda-Suzuki S, Ng J, Tzu J, Dietzl G, Sun Y, Harms M, Nardine T, Luo L, Dickson BJ (2002) Rac function and regulation during Drosophila development. Nature 416:438-442.

Hausen K, Wolburg-Buchholz W, Ribi WA (1980) The synaptic organization of visual interneurons in the lobula complex of flies. A light and electron microscopical study using silver-intensified cobalt-impregnations. Cell Tissue Res 208:371-387.

Hengstenberg R, Hausen K, Hengstenberg B (1982) The number and structure of the giant vertical cells (VS) in the lobula plate on the blowfly Calliphora erythrocephala. J Comp Physiol 149:163-177.

Jalink K, van Corven EJ, Hengeveld T, Morii N, Narumiya S, Moolenaar WH (1994) Inhibition of lysophosphatidate- and thrombin-induced neurite retraction and neuronal cell rounding by ADP ribosylation of the small GTP-binding protein Rho. J Cell Biol 126:801-810.

Jan YN, Jan LY (2001) Dendrites. Genes Dev 15:2627-2641.

Jin Z, Strittmatter SM (1997) Rac1 mediates collapsin-1-induced growth cone collapse. J Neurosci 17:6256-6263.

Kozma R, Sarner S, Ahmed S, Lim L (1997) Rho family GTPases and neuronal growth cone remodelling: relationships between increased complexity induced by Cdc42Hs, Racl, and acetylcholine and collapse induced by RhoA and lysophosphatidic acid. Mol Cell Biol 17: 1201-1211.

Lee T, Luo L (1999) Mosaic analysis with a repressible cell marker for studies of gene function in neuronal morphogenesis. Neuron 22:451-461.

Lee T, Lee A, Luo L (1999) Development of the Drosophila mushroom bodies: sequential generation of three distinct types of neurons from a neuroblast. Development 126:4065-4076.

Lee T, Winter C, Marticke SS, Lee A, Luo L (2000) Essential roles of Drosophila RhoA in the regulation of neuroblast proliferation and dendritic but not axonal morphogenesis. Neuron 25:307-316.

Li Z, Van Aelst L, Cline HT (2000) Rho GTPases regulate distinct aspects of dendritic arbor growth in Xenopus central neurons in vivo. Nat Neurosci 3:217-225.

Luo L (2000) Rho GTPases in neuronal morphogenesis. Nat Rev Neurosci $1: 173-180$.

Luo L (2002) Actin cytoskeleton regulation in neuronal morphogenesis and structural plasticity. Annu Rev Cell Dev Biol 18:601-635.

Luo L, Liao YJ, Jan LY, Jan YN (1994) Distinct morphogenetic functions of similar small GTPases: Drosophila Drac1 is involved in axonal outgrowth and myoblast fusion. Genes Dev 8:1787-1802.

Luo L, Hensch TK, Ackerman L, Barbel S, Jan LY, Jan YN (1996) Differential effects of the Rac GTPase on Purkinje cell axons and dendritic trunks and spines. Nature 379:837-840.

McAllister AK (2000) Cellular and molecular mechanisms of dendrite growth. Cereb Cortex 10:963-973.

Nakayama AY, Harms MB, Luo L (2000) Small GTPases Rac and Rho in the maintenance of dendritic spines and branches in hippocampal pyramidal neurons. J Neurosci 20:5329-5338.

Ng J, Nardine T, Harms M, Tzu J, Goldstein A, Sun Y, Dietzl G, Dickson BJ, Luo L (2002) Rac GTPases control axon growth, guidance and branching. Nature 416:442-447.

Pierantoni R (1976) A look into the cock-pit of the fly. The architecture of the lobular plate. Cell Tissue Res 171:101-122.

Redmond L, Ghosh A (2001) Molecular control of dendritic development. Curr Opin Neurobiol 11:111-117. 
Ridley AJ, Paterson HF, Johnston CL, Diekmann D, Hall A (1992) The smallGTP-binding protein rac regulates growth factor-induced membrane ruffling. Cell 70:401-410.

Ruchhoeft ML, Ohnuma S, McNeill L, Holt CE, Harris WA (1999) The neuronal architecture of Xenopus retinal ganglion cells is sculpted by rhofamily GTPases in vivo. J Neurosci 19:8454-8463.

Scott EK, Luo L (2001) How do dendrites take their shape? Nat Neurosci 4:359-365.

Scott EK, Reuter J, Luo L (2002) Structure of the vertical system and horizontal system neurons of the lobula plate in Drosophila. J Comp Neurol $454: 470-481$

Strausfeld NJ (1976) Atlas of an insect brain. Berlin: Springer.

Tashiro A, Minden A, Yuste R (2000) Regulation of dendritic spine mor- phology by the rho family of small GTPases: antagonistic roles of Rac and Rho. Cereb Cortex 10:927-938.

Threadgill R, Bobb K, Ghosh A (1997) Regulation of dendritic growth and remodeling by Rho, Rac, and Cdc42. Neuron 19:625-634.

Verkhusha VV, Tsukita S, Oda H (1999) Actin dynamics in lamellipodia of migrating border cells in the Drosophila ovary revealed by a GFP-actin fusion protein. FEBS Lett 445:395-401.

Wong WT, Faulkner-Jones B, Sanes JR, Wong ROL (2000) Rapid dendritic remodeling in the developing retina: dependence on neurotransmission and reciprocal regulation by Rac and Rho. J Neurosci 20:5024-5036

Zhang W, Benson DL (2001) Stages of synapse development defined by dependence on F-actin. J Neurosci 21:5169-5181. 